\title{
QUANTUM POTENTIAL AND QUANTUM GRAVITY
}

\author{
J. KOWALSKI-GLIKMAN \\ Institute for Theoretical Physics, University of Wroctaw \\ Pl. Maxa Born 9, 50204 Wroctaw, Poland
}

\begin{abstract}
The quantum potential approach makes it possible to construct a complementary picture of quantum mechanical evolution which reminds classical equation of motion. The only difference as compared to equations of motion for the underlying classical system is the presence of an additional potential term being a functional of the real part of the wavefunction. In the present paper this approach is applied to the quantum theory of gravity based on Wheeler - De Witt equation. We describe the derivation of the 'quantum Einstein equation' and discuss the new features of their solutions.
\end{abstract}

\section{Preface}

It gives me great pleasure to contribute this article to the volume in honor of Professor Jerzy Lukierski. I clearly remember when, still being a student, I have met him for the first time during one of the winter Karpacz schools in late 1970s. Since then he was always close to me, first as an advisor and referee of my $\mathrm{PhD}$ thesis, and then as a close collaborator in many research projects and a very good friend. I cannot possibly fully pay my debt to him; let this article be at least an expression of acknowledgement of what I owe him.

\section{Introduction}

The enigma of quantum gravity is probably the most challenging problem of modern theoretical physics (for the recent reviews see 1,2 , 3 , and 4 ). It is for the difficulty of the problem that in spite of its importance, a very little progress has been made so far. Among many approaches, the canonical quantization seems to be the most natural one for at least two following reasons. First of all, we understand quite well the point we start at: the Einstein theory of general relativity and the Dirac theory of quantization of constrained systems. Secondly, other more exotic approaches (most notably the string theory) introduce, as a rule, a number of their own difficulties.

It is the lesson resulting from the modern developments that the quantum theory of gravity must be non-perturbative. This fact comes from the careful analysis of the condition of diffeomorphism invariance. From this point of view the nonrenormalizibility of perturbative quantum gravity is not surprising; indeed the modern interpretation of this fact is that it just shows that the perturbative expansion of quantum gravity does not make sense.

Most of the recent work an the canonical approach to quantum gravity is related to the loop formulation (see $\mathbf{t}$ and $\mathbf{6}$.) This formulation has the virtue that some part of constraints (Gauss law) is in terms of loop variables solved automatically. However,

*e-mail address: jurekk@ift.uni.wroc.pl and jurekk@fuw.edu.p 
it turned out that this formulation has its own problems, the major of whose are the following:

1. In quantum theory all the composite operators require regularizations. In particular it turns out that in the loop representation the metric is a composite operator and there seems to be no regularization which is consistent with diffeomorphism invariance of the theory. Therefore, even if the theory can be eventually quantized in this representation, it is presumably not equivalent to the quantization in metric representation. (One may argue however that the tetrad representation, which is the starting point for Ashtekar approach, is the more fundamental because only in this representation the coupling to fermions exists).

2. It is well known that contrary to the quantum mechanical case, there exist many representations of quantum field theory which are not equivalent. Thus one must ask the question as to if some prediction of the theory like quantization of volume and area operators 6 , which are claimed to indicate the discrete structure of space-time at sub-Planckian regime, are solid facts and not artifacts of the chosen representation. As a matter of fact, it is unclear if in this formalism one can construct any operator with continuous spectrum at all.

3. One of the major arguments in favor of using Ashtekar variables was the simplicity of the hamiltonian constraints. But it turned out that because of the absence of background metric it is very hard to regularize this operator, and solving the regularized version of it may well be as complicated as it is in the case of the Wheeler-De Witt operator.

I made the comments above just to justify the statement that it is not outrageous to be a little bit old fashioned and base our discussion on the De Witt formulation of quantum gravity 6 . For, if both metric and Ashtekar formulations are equivalent, using them we look at physical reality from two equivalent and yet distinct points of view. If, however, these formulations are not equivalent, they will compete as to which is the correct one. In both cases, therefore, investigating the prediction of metric formulation is quite important. I would like to stress however that the results described below may well be applied to some other formulations of quantum gravity.

Quantum gravity theory faces yet another set of problems. Assume that sooner or later we will have in our possession a class of solutions of this theory. These solutions will be presumably of the form of some wavefunction being a solution of a set of complicated equations defining the theory. Now the question is how should we interpret such a wavefunction? It seems that if we accept the orthodox interpretation of quantum mechanics, we will immediately face a number of problems like: what is the meaning of the wavefunction, how are we to interpret superpositions of states (universes) etc? There are many attempts to address these kind of problems (see e.g., 2).

In my personal opinion it would be fruitful to try to translate the information carried by the wavefunction to the language which would be easier to comprehand. It happens that such a language exists and is provided by the 'pilot wave' or 'quantum potential' approach to quantum mechanics (see 8, 1, 10). Before discussing the merit of this approach as applied to quantum gravity, to set the stage, let us consider some simple examples. 


\section{Quantum potential in action: quantum mechanical examples}

\subsection{The standard quantum mechanical system}

Let us consider the simple one dimensional quantum mechanics. The time evolution of the wave function of particle of mass $m$ is governed by the Schrödinger equation

$$
i \hbar \frac{\partial}{\partial t} \Psi=-\frac{\hbar^{2}}{2 m} \frac{\partial^{2}}{\partial x^{2}} \Psi+V(x) \Psi .
$$

Let us now consider the polar decomposition of the wave function

$$
\Psi(x, t)=R(x, t) \exp \left(\frac{i}{\hbar} S(x, t)\right)
$$

where both $R$ and $S$ are real functions. Substituting this into the Schrödinger equation, we obtain two equations for real and imaginary parts, to wit

$$
\frac{\partial}{\partial t} S+\frac{1}{2 m}\left(\frac{\partial S}{\partial x}\right)^{2}+V(x)-\frac{\hbar^{2}}{2 m} \frac{1}{R} \frac{\partial^{2} R}{\partial x^{2}}=0
$$

and

$$
\frac{\partial}{\partial t} R+\frac{1}{m} \frac{\partial R}{\partial x} \frac{\partial S}{\partial x}+\frac{1}{2 m} R \frac{\partial^{2} S}{\partial x^{2}}=0
$$

The interpretation of Eq. (3) is clear: this is noting but the continuity equation. Eq. (2), on the other hand, can be interpreted as the Hamilton-Jacobi equation, with the additional term proportional to $\hbar^{2}$ and resulting from the wave function of the system. This potential is called the 'quantum potential' and it is customary to denote it by $Q(x, t)$. Observe that this potential is, in general, time dependent. It is a well known fact that the Hamilton-Jacobi equation contains the whole dynamics of the system. Thus, interpreting Eq. (2) in such a way, we can immediately write Hamilton equations of motion:

$$
\begin{aligned}
& \dot{p}=\{p, \mathcal{H}\}, \\
& \dot{x}=\{x, \mathcal{H}\},
\end{aligned}
$$

where

$$
\mathcal{H}=\frac{p^{2}}{2 m}+V(x)-\frac{\hbar^{2}}{2 m} \frac{1}{R} \frac{\partial^{2} R}{\partial x^{2}}
$$

is the effective hamiltonian containing the quantum potential term.

Let us discuss interpretation of these equations. To set the stage, let us consider Schrödinger equation first. Here we have to provide one initial condition for $\Psi$, $\Psi_{0}=\Psi(x, t=0)$ which corresponds to two initial conditions for $R$ and $S$. It should be observed that Eq.\% (3) expresses conservation of probability.

Now, in the quantum potential approach the situation is quite different. First of all, Hamilton's equations (14), (5) govern the evolution of $x(t), p(t)$. We need therefore two initial conditions for $p$ and $x$. Second, we have Eq. (3) which shapes time evolution of $R$ and therefore the time dependence of quantum potential. The initial condition for this equation plays double role. First, $R^{2}(x, 0)$ is to be interpreted 
as the probability distribution for various initial conditions for $x$, and second $R(x, 0)$ is itself an initial condition for $R$ time evolution.

This shows that in the case of this equation we have to do with some kind of personality split. To cure this disease, we should, in principle, proceed as follows.

Recall that $\frac{\partial S}{\partial x}=p$. Thus, Eq. (3) can be rewritten as

$$
\frac{\partial}{\partial t} R+\frac{1}{m} \frac{\partial R}{\partial x} p+\frac{1}{2 m} R \frac{\partial^{2} S}{\partial x^{2}}=0
$$

If we could express $\frac{\partial^{2} S}{\partial x^{2}}$ as a function of $p, q, t$, we would be able to turn the equations governing the quantum potential dynamics to closed form. Moreover, we will be able manifestly take into account the back reaction of $p$ and $x$ on the quantum potential itself. This procedure is currently under investigation, and in this paper I will ignore this equation whatsoever assuming that it is identically satisfied.

To finish this subsection let me make some remarks.

The dynamical equations above describe trajectory of a particle. One may treat this trajectory as some unphysical (i.e., not related to reality) auxiliary 'picture' of the particle behavior. This point of view is especially fruitful in the cases when in terpretation of the wave function is not clear for example in quantum cosmology 11. However, as stressed by Bohm and Hiley in 8, one may take the 'ontological' stand and assume that the trajectories are real, that is that there exists a 'real' particle moving along the trajectories. It should be mentioned that even if such an interpretation of quantum mechanics contains 'hidden variables' (the trajectory itself) it does not contradict the Bell theorem $\mathbf{9}$, because the theory is clearly non-local. This fact is not by itself very much fearsome since, as careful analysis of Einstein - Podolsky Rosen paradox shows, in the standard Bohr - Von Neumann interpretation of quantum mechanics, the theory is non-local as well.

Let me now turn to more complex example, which I will call

\subsection{Cosmo quantum mechanics}

In this subsection I will consider an example of the so-called one dimensional parametric systems. Systems like that possess a symmetry which makes them independent of a parametrization of the world line; this symmetry is the one dimensional diffeomorphism invariance which is analogous to the diffeomorphism invariance in four dimensions. On the classical level the whole dynamics of the parametric particle is given by one constraint being the hamiltonian; in quantum case, according to the Dirac method of quantization of constrained systems, the hamiltonian operator must annihilate physical state, and thus the wave function is, by virtue of the Schrödinger equation, time independent.

Now consider a parametric model with simple hamiltonian (which nevertheless is of importance in quantum cosmology, see 11$)^{\dagger}$

$$
\mathrm{H} \Psi\left(x^{i}\right)=\left(\frac{1}{2} g^{i j} \nabla_{i} \nabla_{j}-V\left(x^{i}\right)\right) \Psi\left(x^{i}\right)=\left(\frac{1}{2} \square-V\left(x^{i}\right)\right) \Psi\left(x^{i}\right)=0,
$$

${ }^{\dagger}$ In what follows all quantum mechanical operators will be written in sans serif type face: $a, b, \ldots$, A, B etc. 
where $g^{i j}$ may be $x$-dependent. Let us assume again that $\Psi$ has the following polar decomposition

$$
\Psi=R\left(x^{i}\right) \exp \left(\frac{i}{\hbar} S\left(x^{i}\right)\right)
$$

with both $R$ and $S$ real. Inserting (8) into (17), we obtain two equations corresponding to real and imaginary part, respectively. These equations read

$$
\begin{gathered}
\mathcal{H}[S(x)]=\frac{1}{2} g_{i j} \frac{\partial S}{\partial x^{i}} \frac{\partial S}{\partial x^{j}}+V\left(x^{i}\right)=\frac{\hbar^{2}}{2} \frac{1}{R} \square R, \\
R \square S+2 g^{i j} \frac{\partial S}{\partial x^{i}} \frac{\partial R}{\partial x^{j}}=0 .
\end{gathered}
$$

Equation (10) will not concern us anymore. As in the previous example it corresponds to probability conservation. We assume that the wave function $\Psi$ is a solution of equation (7), and thus this equation is identically satisfied (even though we may not know what the explicit form of the wavefunction is.) On the other hand, equation (9) is of crucial importance. This equation can be used to derive the time dependence and then serves as the evolutionary equation in the formalism.

For, let us introduce time $t$ through the following equation

$$
\frac{d x^{i}}{d t}=g^{i j} \frac{\delta \mathcal{H}[S(x)]}{\delta\left(\partial S / \partial x^{j}\right)} .
$$

This equation defines the trajectory $x^{i}(t)$ in terms of the phase of the wavefunction $S$. Now we can substitute back equation (11) to (9). Assuming that the matrix $g^{i j}$ has the inverse $g_{i j}$, we find $\left(\dot{x}^{i}=\frac{d x^{i}}{d t}\right)$

$$
\frac{1}{2} g_{i j} \dot{x^{i} \dot{x}^{j}}+V\left(x^{i}\right)=\frac{\hbar^{2}}{2} \frac{1}{R} \square R .
$$

We see therefore that the quantum evolution differs from the classical one only by the presence of the quantum potential term

$$
-V_{\text {quant }}\left(x^{i}\right)=\frac{\hbar^{2}}{2} \frac{1}{R} \square R
$$

on the right hand side of equation of motion. Since we assume that the wave function is known, the quantum potential term is known as well.

Equation (12) is not in the form which is convenient for our further investigations. To obtain the desired form, we define classical momenta

$$
p_{i}=\frac{\delta \mathcal{H}[S(x)]}{\delta\left(\partial S / \partial x^{i}\right)}=g_{i j} \dot{x}^{j}
$$

and cast equation (12) to the form

$$
\mathcal{H} \equiv \frac{1}{2} g^{i j} p_{i} p_{j}+V\left(x^{i}\right)-\frac{\hbar^{2}}{2} \frac{1}{R} \square R=0 .
$$


We regard $\mathcal{H}$ as the generator of dynamics acting through the Hamilton equations

$$
\begin{aligned}
\dot{p}_{i} & =-\frac{\partial \mathcal{H}}{\partial x^{i}} \\
\dot{x}^{i} & =\frac{\partial \mathcal{H}}{\partial p_{i}} .
\end{aligned}
$$

The time evolution is therefore governed by equations (14) subject to the constraint for initial conditions (13). This completes the technical part. However a number of remarks is in order.

1. The quantum potential interpretation may be used to obtain a well defined semi-classical approximation to quantum theory. Indeed, it can be said that the system enters the (semi-) classical regime if the quantum potential is much smaller than the regular potential term.

2. One of the major advantages of the quantum potential approach is that it provides one with an affective and simple way of introducing time even if the system under consideration has a hamiltonian as one of the constraints. In particular this approach serves as a possible route to final understanding of the problem of time in quantum gravity.

3. Related to this is the problem of interpretation of wave functions which are real. This problem has been a subject of numerous investigations, but from the point of view of quantum potential the resolution of it is quite simple. We just say that real wavefunctions (of the universe) represent a model without time evolution (and therefore time) at all. ${ }^{\ddagger}$ It is clear from the formalism: $\dot{x}$ is just equal to zero, so nothing evolves and therefore there is no clock to measure time. On the other hand, equation (12) means that for the real wave function the system settles down to the configuration for which the total potential (i.e., classical plus quantum) is equal to zero.

But there is one important modification of the theory for real wave functions. Such wave functions, which form a degenerate subclass of all wave functions being solutions of the theory, should be regarded as ones which impose additional constraints on the quantum potential theory, namely that the momentum is zero. This constraint, together with $\mathcal{H}=0$ form a second class system. This means, first, that one can take them as strong equalities (up to the standard manipulations with Dirac bracket), and this leads to the abovementioned equality of classical and quantum potential. What is more important, however, since the hamiltonian is now a second class constraint, it does not generate gauge transformations (time reparametrization) any more. Roughly speaking, the quantum potential theory is "anomalous" for real wave functions. It should be mentioned that similar effect turns out to be present in the full quantum gravity theory, and without the interpretation given above it may lead to apparent paradoxes, one of whose was presented in 22 . I will discuss the quantum gravity case below.

4. The definition of time by equation (11) is, of course, not unique. In fact, we can use a more general expression

$$
\dot{x}^{i}=N(t) \frac{\delta \mathcal{H}[S(x)]}{\delta\left(\partial S / \partial x^{i}\right)},
$$

\footnotetext{
${ }^{\ddagger}$ Recall that the standard (time-dependent) Schrödinger equation does not have any real solutions.
} 
where, in the case of gravity, the function $N$ is to be identified with the lapse function of ADM formalism.

\section{Wheeler - De Witt equation and its formal solution}

Now we can turn to the real thing - the quantum theory of gravity. This theory is defined by two sets of constraints, one of whose are generators of three dimensional diffeomorphisms operators:

$$
\mathrm{H}_{a}=-2 \nabla_{b} \mathrm{p}_{a}^{b}
$$

and the second consists of a single but very complicated operator, called the Wheeler - De Witt operator

$$
\mathrm{H}_{W D W}=\frac{1}{2 \mu} \mathcal{G}_{a b c d} \mathrm{p}^{a b} \mathrm{p}^{c d}+\mu \sqrt{h}\left(2 \Lambda-{ }^{(3)} \mathcal{R}\right) .
$$

Here $\mu=(16 \pi G)^{-1}, G$ is the Newton's constant,

$$
\mathcal{G}_{a b c d}=\frac{1}{\sqrt{h}}\left(h_{a c} h_{b d}+h_{a d} h_{b c}-h_{a b} h_{c d}\right)
$$

$\mathrm{p}^{a b}$ are momentum operators related to the three metric $h_{a b},{ }^{(3)} \mathcal{R}$ is the three dimensional curvature scalar, and $\Lambda$ the cosmological constant.

As it stands, this operator is meaningless. It contains the product of two functional derivatives acting at the same point; as a result, while acting on a wave function, the product of two delta functions at the same point appears. This is, of course standard property of second order operators in Schrödinger representation. The way out of this problem is to regularize the kinetic term of the operator, that is, to replace $\mathrm{H}_{W D W}$ above by

$$
\begin{gathered}
\mathrm{H}_{W D W}^{r e g}(x ; t)=\lim _{x \rightarrow x^{\prime}}\left\{\frac{1}{2 \mu} \mathcal{K}_{a b c d}\left(x, x^{\prime} ; t\right) \mathrm{p}^{a b}(x) \mathrm{p}^{c d}(y)\right\}+\mu \sqrt{h}\left(2 \Lambda-{ }^{(3)} \mathcal{R}\right)= \\
=\frac{1}{2 \mu} \triangle_{r e g}+\mu \sqrt{h}\left(2 \Lambda-{ }^{(3)} \mathcal{R}\right)
\end{gathered}
$$

where in the limit when the parameter $t$ goes to zero,

$$
\lim _{t \rightarrow 0} \mathcal{K}_{a b c d}\left(x, x^{\prime} ; t\right)=\mathcal{G}_{a b c d}(x) \delta\left(x-x^{\prime}\right) .
$$

The function $\mathcal{K}$ can be found, for example, by using the heat kernel equation

$$
\frac{\partial}{\partial t} \mathcal{K}\left(x, x^{\prime} ; t\right)=\nabla_{\left(x^{\prime}\right)}^{2} \mathcal{K}\left(x, x^{\prime} ; t\right)
$$

with the initial condition (20). This approach was proposed in 13 in the context of Yang - Mills theory, and then applied to the theory of gravity in 14 . In this latter paper the action of the regularized WDW operator on some simple expressions built from 
the metric and curvature was also found. The virtue of this approach is that mathematically meaningless expressions (like products of delta functions at the same point) are now replaced by well controllable singularities of $\mathcal{K}(x ; t)=\lim _{x \rightarrow x^{\prime}} \mathcal{K}\left(x, x^{\prime} ; t\right)$ for small $t$. Each singular term can be then replaced by a renormalization constant. The problem is however that at the first glance it seems that the number of such independent constants is infinite. However, the action of the operator has been tested on some simple expressions (see 14 ) and one may hope that the physical wave functions, i.e., the wavefunctions annihilated by all constraints (or, to be more precise, the matrix elements constructed with the help of them) will eventually contain only finite number of them (hepefully two - one corresponding to the renormalization of the Newton's constant, and second to the renormalization of the cosmological constant. $)^{\S}$

Be as it may, let us assume that we have constructed the regularized Wheeler - De Witt operator. Then one can easily write down the formal expression for the physical wave function being annihilated by this operator. This can be done by observing that a physical state is of the form

$$
\Psi_{\text {phys }}=\delta\left(\mathrm{H}_{W D W}^{r e g}\right) \psi[h],
$$

where $\psi[h]$ is any wave function(al) of the metric, that is

$$
\Psi_{\text {phys }}=\int \mathcal{D} N(x) e^{i \int d^{3} x N(x) \mathrm{H}_{W D W}^{r e g}(x)} \psi[h] .
$$

Even though the expression above is very formal, it can be used to obtain a perturbative expansion (in $\mu$ ) of a solution. Second, and this will be quite important in our analysis below, using this formula we can find out some properties of the physical wave function.

The next important property of the formula (23) is that it may be used as the starting for construction of the inner product. It should be recalled that one of the major problem of the Dirac quantization procedure is that it does not offer any constructive way of finding what the inner product of physical states is. I will not dwell on this problem any longer as it is the subject of the forthcoming paper 15 . Let me only mention that some partial results and discussion are contained in 11 .

Now we are ready to extend the discussion of one dimensional parametric systems presented in subsection 3.1 to the case of full theory of gravity. This will by the subject of the next section.

\section{Quantum potential for quantum gravity}

As in subsection 3.1 we start with decomposition of the wave function to the polar form (from now on $\hbar=G=c=1$ )

$$
\Psi[h]=R[h] e^{i S[h]} .
$$

§I would like to thank Jeff Greensite for long discussion concerning this point.

The commutator of two WDW operators is proportional to the diffeomorphism operators (see 14 for discussion of the algebra of regularized constraints in quantum gravity.) Therefore it is not needed to apply diffeomorphism constraints separately. 
Substituting this into the WDW equation, one easily finds (see also 12 )

$$
-\frac{1}{2 \mu} \mathcal{K}_{a b c d} \frac{\delta S}{\delta h_{a b}} \frac{\delta S}{\delta h_{c d}}+\mu \sqrt{h}\left(2 \Lambda-{ }^{(3)} \mathcal{R}\right)+\frac{1}{2 \mu} \frac{\triangle_{\text {reg }} R}{R}=0 .
$$

If one identifies momenta with the (functional) gradient of $S$, to wit

$$
p^{a b}(x)=\frac{\delta S}{\delta h_{a b}}(x)
$$

this equation turns to the Hamilton-Jacobi equation for general relativity

$$
-\frac{1}{2 \mu} \mathcal{G}_{a b c d} p^{a b} p^{c d}+\mu \sqrt{h}\left(2 \Lambda-{ }^{(3)} \mathcal{R}\right)+\frac{1}{2 \mu} \frac{\triangle_{r e g} R}{R}=0
$$

with the additional last term corresponding to quantum potential. This equation (without the potential term, of course) was first analyzed by Gerlach 16. Equation (26) is only one of two equations which result from action of the WDW operator on the wave function. As in the case of the parametric particle mechanics, we ignore the second equation, which could be interpreted as an equation shaping the quantum potential and/or guaranteeing conservation of probability.

The wave function is subject to the second set of equations, namely the ones enforcing the three dimensional diffeomorphism invariance. These equations read (after decomposing into real and imaginary part)

$$
\begin{gathered}
\nabla^{a} \frac{\delta S}{\delta h_{a b}}=\nabla^{a} p_{a b}=0 \\
\nabla_{a} \frac{\delta R}{\delta h_{a b}}=0
\end{gathered}
$$

Each of these equations has different interpretation. The second expresses invariance of $R$ with respect to spatial diffeomorphisms - this invariance is actually guaranteed by the fact that $R$ is the modulus of a wave function being a solution o WDW equation; the first is to be interpreted as a constraint equation.

Thus our theory is defined by two equations (26) and (27). Now we can follow without any alternations the derivation of Gerlach 16 to obtain the full set of ten equations governing the quantum gravity theory in quantum potential approach

$$
\begin{aligned}
0=\mathcal{H}^{a} & =\nabla_{a} p^{a b}, \\
0=\mathcal{H}_{\perp} & =-\frac{1}{2 \mu} \mathcal{G}_{a b c d} p^{a b} p^{c d}+\mu \sqrt{h}\left(2 \Lambda-{ }^{(3)} \mathcal{R}\right)+\frac{1}{2 \mu} \frac{\triangle_{r e g} R}{R} \\
\dot{h}_{a b}(x, t) & =\left\{h_{a b}(x, t), \mathcal{H}[N, \vec{N}]\right\} \\
\dot{p}^{a b}(x, t) & =\left\{p^{a b}(x, t), \mathcal{H}[N, \vec{N}]\right\} .
\end{aligned}
$$

In equations above, $\{\star, \star\}$ is the usual Poisson bracket, and

$$
\mathcal{H}[N, \vec{N}]=\int d^{3} x\left(N(x) \mathcal{H}_{\perp}(x)+N^{a}(x) \mathcal{H}_{a}(x)\right)
$$

is the total hamiltonian (which is a combination of constraints).

A number of comments is in order 
1. Let us observe that we somehow "solved" the problem of time in quantum general relativity. Indeed out of sudden time appears in Eqs. (31), (32). It is a matter of taste if this is to be considered as a solution of this problem, nevertheless, in this formulation the problem of time in quantum general relativity is as simple (or as difficult) as the analogous problem in classical theory of gravitation (see 目.)

2. The very important problem related to Eqs. (29-32) is under which conditions these equations are equivalent to ten four dimensional covariant equations ("quantum Einstein equations".) To see what this problem is about, let us observe that Eqs. (29), (30) are constraint equations. This means that $\mathcal{H}_{a}$ and $\mathcal{H}_{\perp}$ must have (weakly) vanishing Poisson bracket with $\mathcal{H}[N, \vec{N}]$. But this means that Poisson brackets of $\mathcal{H}_{a}$ and $\mathcal{H}_{\perp}$ must form closed algebra. Yet it is well known that it is quite difficult to close the bracket

$$
\left\{\mathcal{H}_{\perp}(x), \mathcal{H}_{\perp}(y)\right\}
$$

for arbitrary quantum potential. Indeed one can easily check that this Poisson bracket does not close if $\mathcal{H}_{\perp}$ contains, for example, terms quadratic in curvature." Now, a simple inspection of Eq. (23) shows that $\Psi_{\text {phys }}$ will contain higher order terms. It should be observed however that quantum potential term $\frac{1}{2 \mu} \frac{\triangle_{\text {reg }} R}{R}$ is essentially non local and the standard argument may be not applicable. This problem will be the subject of the separate paper.

Assuming however that for some quantum potentials the bracket above does not close. Then we have to do with secondary constraints which will be second class (this follows from simple counting of degrees of freedom.) This just means that the $3+1$ symmetry is not promoted to the full four dimensional diffeomorphism invariance after quantization. This fact is without doubts very important, for example it may be related to the appearance of minimal length in quantum gravity (see 17). It may also mean that either $3+1$ splitting being the first step of quantization procedure is incorrect and/or that the four dimensional diffeomorphism invariance is just a low-energy phenomenon.

3. Talking anomalies, let us return to the apparent puzzle discussed in 12 . In this paper the author argues that for real solution of WDW equation there exists the transformation generated by the hamiltonian $\mathcal{H}_{\perp}$ which transforms the solution of Eqs. (31), (32) into configuration which is not a solution. But if the wave function we start with is real, the momentum of gravitational field vanishes. Then the quantum potential must compensate the classical "potential term" the three-curvature. As in the case of parametric particle, this means that the constraint $\mathcal{H}_{\perp}$ together with $p^{a b}$ becomes a second class system and the time shift invariance of the theory is lost.

4. Let me stress once again that one of the crucial points of the construction is an appropriate regularization of the kinetic (derivative) term in $\mathcal{H}_{\perp}$. This problem is not discussed in most papers concerning (semiclassical) quantum gravity in metric representation. However without proper understanding of regularization

"This fact can be understood by observing that the effective theory in four dimensions is, in this case, a higher derivative theory. 
and renormalization of the theory it is impossible to find any solution and to even start the program described above.

5. Finally, it is feasible to extend the above discussion to the case of Ashtekar variable (in either connection or tetrad representation.) This is especially important because one clearly should include matter fields and it is well known that we need tetrad formalism to take care of fermions (for bosonic matter fields there are only obvious technical modifications of the formalism.)

To conclude, the qunatum potential program may provide us with an important insight into the meaning of the physical wave function of quantum gravity. However, we need to find a wave function of the universe first. As for now, therefore, this set of ideas can be only applied in the context of the minisuperspace models. Let us hope that this situation will soon change.

\section{Acknowledgement}

I would like to thank Prof. Jeff Greensite and Mr. Arkadiusz Błaut for discussions concerning the problems discussed in this paper.

\section{Bibliography}

1. C. J. Isham in Canonical Quantum Gravity: from Classical to Quantum, Springer Verlag, Berlin, 1994.

2. C. J. Isham in Integrable Systems, Quantum Groups, and Quantum Field Theories, L. A. Ibort and M. A. Rodriguez eds., Kluwer Academic Publishers, Dordrecht,1993.

3. C. J. Isham Structural Issues in Quantum Gravity, GR14 plenary session lecture, gr-qc 9510063.

4. A. Ashtekar, Non-Perturbative Canonical Quantum Gravity, World Scientific, Singapore, 1991.

5. Class. Quant. Grav. 8, 1613 (1991).

6. Nucl. Phys. B 442, 593, (1995)

7. Bryce S. De Witt, Phys. Rev. 160, 1113, 1967.

8. D. Bohm, Phys. Rev. 85, 166, (1952), Phys. Rev. 85, 180, (1952); D. Bohm and B.J. Hiley Phys. Rep. 144, 323, (1987), D. Bohm, B.J. Hiley, and P.N. Koloyerou Phys. Rev. 144, 349, (1987); D. Bohm, B.J. Hiley, The Undivided Universe: An Ontological Interpretation of Quantum Theory, Routledge \& Kegan Paul, London, 1993.

9. J. S. Bell Speakable and Unspeakable in Quantum Mechanics, Cambridge University Press, 1987.

10. K. Berndl, M. Daumer, D. Dürr, S. Goldstein, N. Zanghi, A Survey of Bohmian Mechanics, to appear in Il Nuovo Cimento and references therein.

11. A. Błaut and J. Kowalski-Glikman, gr-qc 9509040, to appear in Class. Quant. Grav.

12. Yu. Shtanov Pilot Wave Quantum Gravity, gr-qc 9503005

13. Paul Mansfield, Nucl. Phys. B 418, 113 (1994).

14. T. Horiguchi, K. Maeda, M. Sakamoto, Phys. Lett. 344 B, 105, (1995).

15. J. Kowalski-Glikman On the Inner Product for Dirac Quantization Scheme, in preparation. 
16. Ulrich H. Gerlach, Phys. Rev. 177, 1929 (1969).

17. L. J. Garay, Int. J. Mod. Phys. A 10, 145 (1995). 